\title{
Model-based Analysis of myocardial strains in Left Bundle Branch Block Patients
}

\author{
Kimi P. Owashi ${ }^{1}$, Elena Galli ${ }^{1}$, Arnaud Hubert ${ }^{1}$, Erwan Donal ${ }^{1}$, Alfredo I. Hernandez ${ }^{1}$ and \\ Virginie Le Rolle ${ }^{1}$ \\ ${ }^{1}$ Univ Rennes, Inserm, LTSI - UMR 1099, F-35000 Rennes, France
}

\section{Background}

Although patients with typical left bundle branch block (LBBB) show greater efficacy of cardiac resynchronization therapy (CRT), there is a wide range of responses to CRT with a subset of patients showing little or no improvement. Recently, it has been shown that combination of echo-based parameters of LV dyssynchrony and QRS duration helped to improve the prediction of the response to CRT. However, the interpretation of features extracted from echocardiography could be difficult due the complexity of mechanisms involved in cardiac contraction. Computational modelling could help to improve the interpretation of myocardial strains in LBBB patients before implantation.

Aims of the study is to propose a patient-specific model-based approach in order to assist the analysis of myocardial strains and to improve the interpretability of echo-based parameters.

\section{Methods}

The proposed model of the cardiovascular system integrates four main sub-models: 1) cardiac electrical system, 2) right and left atrium, 3) multi-segment right and left ventricles and 4) systemic and pulmonary circulations. Patient-specific simulations of myocardial strains were obtained by coupling the computational model and the clinical data to a parameter identification algorithm. The proposed approach was evaluated on data obtained from 10 LBBB patients, including ischaemic $(n=5)$ and non-ischaemic $(n=5)$ cardiomyopathy. Bull's-eye representations of the electrical activation time and contractility levels were analysed.

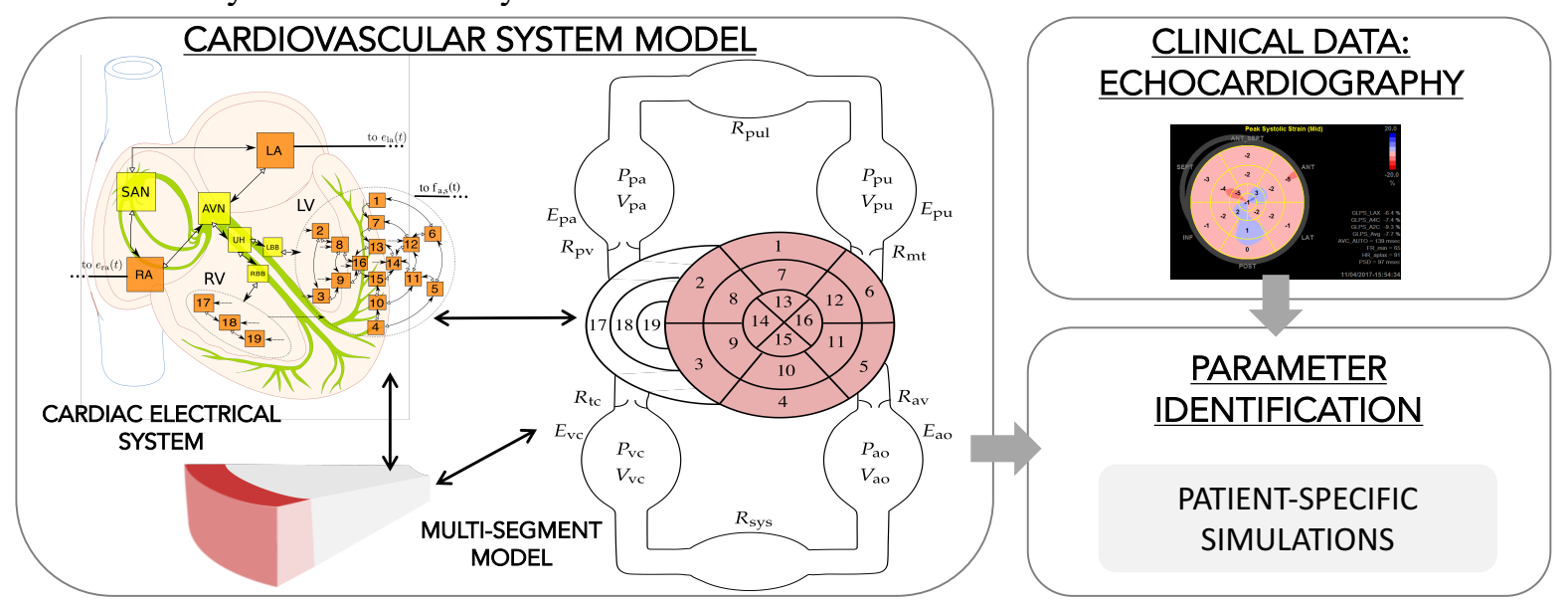

Figure 1. Patient-specific simulation based on a parameter identification algorithm.

\section{Results}

The comparison between simulated and experimental strains for the 10 subjects reflects a satisfying adaptation of the model to different strain morphologies. The mean errors between real and synthesized signals are equal to $3.7168 \pm 0.9075$. Fig. 2 presents simulated (black line) and experimental (red line) myocardial strain signals for selected LBBB patients with a lateral ischaemia, anterior ischaemia and non-ischaemia. The bull's-eye representations of electrical activation delay and contractility levels, estimated from the model-based approach, for ischemic and non-ischemic LBBB is included in the figure. Contractility bull's-eye results allow ischaemic and non-ischaemic cases distinction, where low levels of contractility could be associated with damaged tissues. 


\section{Ischaemic IML LBBB}
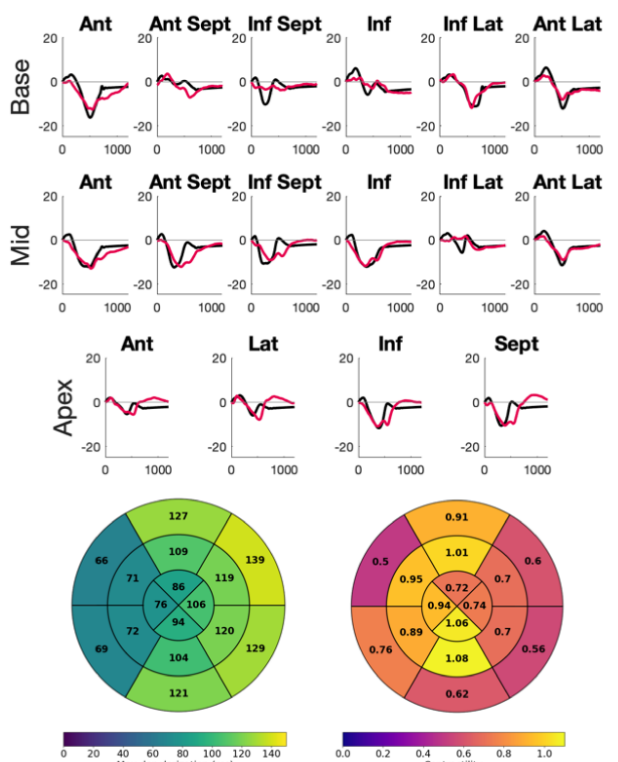

Ischaemic IMA LBBB
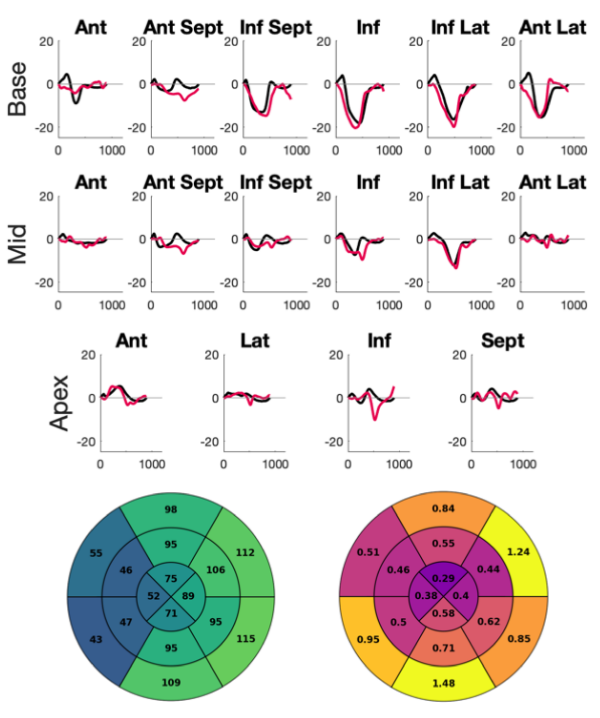

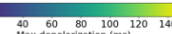

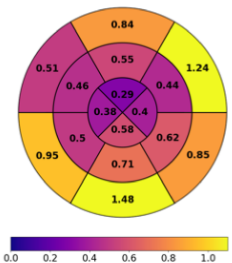

Non-Ischaemic LBBB

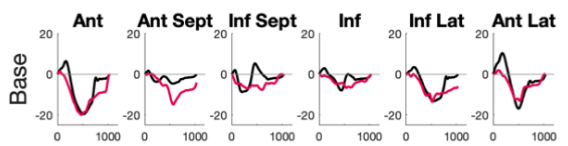

Ant ${ }_{20}^{\text {Ant Sept }}{ }_{20}$ Inf Sept ${ }_{20}$ Inf ${ }_{20}$ Inf Lat ${ }_{20}$ Ant Lat

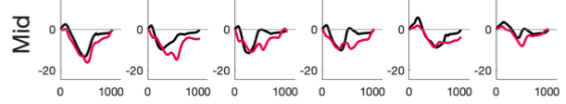

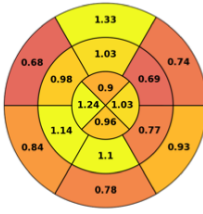

40
Max cepolarzation (ms)
(m)

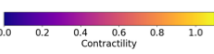

Figure 2. Myocardial strain signals obtained from clinical data (red) and patient-specific simulations (black). Bull's-eye representations of the segmental activation delay (left) and contractility (right) for a lateral ischemic (left), anterior ischemic (middle) and non-ischemic (right) patient.

\section{Conclusion}

Identified parameters show significant electrical conduction and mechanical activation delays for the LBBB patient and hypocontractile myocardial segments were successfully localized from the modelbased approach. 